\title{
Modelling the thermal X-ray emission around the Galactic centre from colliding Wolf-Rayet winds
}

\section{Christopher M. P. Russell ${ }^{1}$, Q. Daniel Wang ${ }^{2}$ and Jorge Cuadra ${ }^{3}$}

\author{
${ }^{1}$ X-ray Astrophysics Laboratory, NASA/Goddard Space Flight Center, \\ Greenbelt, MD 20771, USA (NASA Postdoctoral Program Fellow, administered by USRA) \\ email: crussell@udel.edu \\ ${ }^{2}$ Department of Astronomy, University of Massachusetts, Amherst, MA 01003, USA \\ ${ }^{3}$ Instituto de Astrofísica, Facultad de Física, Pontificia Universidad Católica de Chile, \\ 782-0436 Santiago, Chile
}

\begin{abstract}
The Galactic centre is a hotbed of astrophysical activity, with the injection of wind material from $\sim 30$ massive Wolf-Rayet (WR) stars orbiting within $12^{\prime \prime}$ of the super-massive black hole $(\mathrm{SMBH})$ playing an important role. Hydrodynamic simulations of such colliding and accreting winds produce a complex density and temperature structure of cold wind material shocking with the ambient medium, creating a large reservoir of hot, X-ray-emitting gas. This work aims to confront the 3Ms of Chandra X-ray Visionary Program (XVP) observations of this diffuse emission by computing the X-ray emission from these hydrodynamic simulations of the colliding WR winds, amid exploring a variety of SMBH feedback mechanisms. The major success of the model is that it reproduces the spectral shape from the $2^{\prime \prime}-5^{\prime \prime}$ ring around the SMBH, where most of the stellar wind material that is ultimately captured by $\mathrm{Sgr} \mathrm{A}^{*}$ is shock-heated and thermalised. This naturally explains that the hot gas comes from colliding WR winds, and that the wind speeds of these stars are in general well constrained. The flux level of these spectra, as well as $12^{\prime \prime} \times 12^{\prime \prime}$ images of $4-9 \mathrm{keV}$, show the X-ray flux is tied to the SMBH feedback strength; stronger feedback clears out more hot gas, thereby decreasing the thermal X-ray emission. The model in which Sgr A* produced an intermediate-strength outflow during the last few centuries best matches the observations to within about 10\%, showing SMBH feedback is required to interpret the $\mathrm{X}$-ray emission in this region.
\end{abstract}

Keywords. Galaxy: centre, stars: Wolf-Rayet, stars: winds, outflows, X-rays: stars, radiative transfer, hydrodynamics

\section{Introduction}

The proximity of Sgr A* makes it the only SMBH where its orbiting stars are resolved, and therefore is the best opportunity to study the interplay between a SMBH and the stars and ejected wind material orbiting it. Two examples of work in this thread are the hydrodynamic simulations of the winds of 30 Wolf-Rayet (WR) stars orbiting Sgr A* (Cuadra et al. 2008, 2015), and the $3 \mathrm{Ms}$ of Chandra X-ray Visionary Program (XVP) observations (Wang et al. 2013) that resolved, among other things, the diffuse thermal emission around $\mathrm{Sgr} \mathrm{A}^{*}$ thought to originate from wind-wind collisions.

This work computes the thermal X-ray emission from the aforementioned hydrodynamics simulations and compares the results to the XVP observations with the aim of increasing our understanding of the WR stars, and more generally the full environment, surrounding Sgr A*. The full version of this work is Russell, Wang \& Cuadra (2016). 


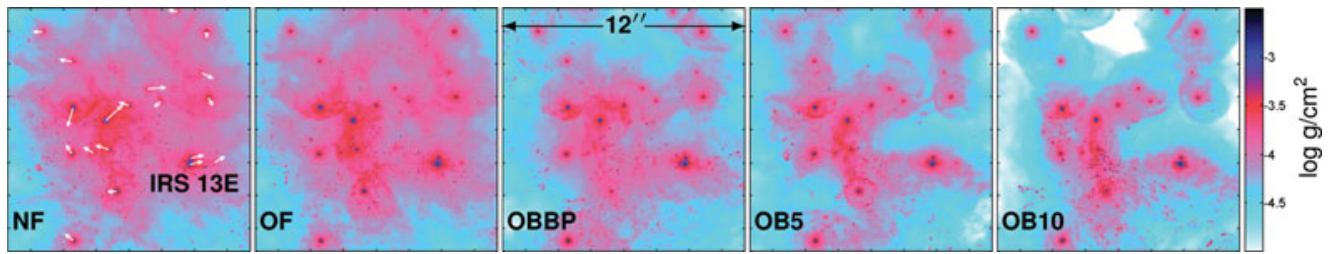

Figure 1. Column density of the central $12^{\prime \prime} \times 12^{\prime \prime}$ for all models with the strength of the SMBH feedback increasing from left to right. The arrows indicate the projected velocities of the stars. The tick marks are every $2 \times 10^{17} \mathrm{~cm}$, and the IRS $13 \mathrm{E}$ cluster is labeled.

Table 1. Left: Parameters of SMBH feedback for each model. Center: Results of the ISM absorption fitting over the entire spectrum of the $2^{\prime \prime}-5^{\prime \prime}$ ring (excluding IRS13E and the PWN), showing the inverse norm (i.e. [model flux]/[observed flux]), $N_{\mathrm{H}}$ with $90 \%$ confidence interval errors, and $\chi_{\text {red }}^{2}$ for each model. Each fit has 211 dof. Right: Model-to-data ratio of the $4-9 \mathrm{keV}$ flux from $2^{\prime \prime}-5^{\prime \prime}$ ring (excluding IRS13E and the PWN). The differences in the image and spectral results account for the different X-ray background estimations for each observable, resulting in the observed image flux being an upper limit and the observed spectral flux being a lower limit.

\begin{tabular}{l|ccc|ccc|ccc} 
model & $\begin{array}{c}\dot{M}_{\text {out }} \\
\left(M_{\odot} / \mathrm{yr}\right)\end{array}$ & $\begin{array}{c}v_{\text {out }} \\
(\mathrm{km} / \mathrm{s})\end{array}$ & direction & $\begin{array}{c}\text { norm } \\
(\mathrm{mod} / \mathrm{obs})\end{array}$ & $\begin{array}{c}N_{\mathrm{H}} \\
\left(10^{22} \mathrm{~cm}^{-2}\right)\end{array}$ & $\begin{array}{c}\chi_{\text {red }}^{2} \\
\text { image spectra mean } \\
\text { (model/observed) }\end{array}$ \\
\hline NF & 0 & 0 & - & 1.95 & $10.28_{-0.28}^{+0.29}$ & 1.36 & 1.50 & 1.97 & 1.73 \\
OF & $\dot{M}_{\text {in }}$ & 10,000 & spherical & 1.94 & $10.26_{-0.28}^{+0.29}$ & 1.36 & 1.50 & 1.96 & 1.72 \\
OBBP & $10^{-4}$ & 5,000 & bipolar, $15^{\circ}$ & 1.20 & $10.16_{-0.27}^{+0.29}$ & 1.38 & 0.92 & 1.21 & 1.07 \\
OB5 & $10^{-4}$ & 5,000 & spherical & 1.17 & $10.92_{-0.28}^{+0.30}$ & 1.31 & 0.92 & 1.20 & 1.06 \\
OB10 & $10^{-4}$ & 10,000 & spherical & 0.79 & $10.73_{-0.29}^{+0.31}$ & 1.24 & 0.62 & 0.81 & 0.71
\end{tabular}

\section{Method}

Fig. 1 shows the column density at the present day for all hydrodynamic simulations (Cuadra et al. 2008, 2015). As the feedback strength increases from left to right, the amount of material remaining in the simulation volume decreases. The model names are $\mathrm{NF}$ - no feedback, OF - outflow, OBBP - bipolar outburst, OB5 - 5,000 km/s outburst, and OB10 - 10,000 km/s outburst. All outbursts occur from 400 to $100 \mathrm{yr}$ ago (Ponti et al. 2010). Table 1 (left) provides more details of each model.

We solve the formal solution of radiative transfer along a $500 \times 500$ grid of rays covering the central $15^{\prime \prime} \times 15^{\prime \prime}$ through the simulation volume for $0.3-12 \mathrm{keV}$ (covering the Chandra HETG response function) at a resolution of 800 energy bins per dex. These pixel maps are folded through the Chandra ACIS-S/HETG 0th-order response function to compare with the observed spectrum, and then through the Chandra PSF (which is approximated as a $0.5^{\prime \prime}$ full-width half-max [FWHM] Gaussian) to compare with the observed image.

The basis of the radiative transfer calculation (see Russell 2013; Russell et al. 2016 for more details) is the SPH visualization program SPLASH (Price 2007). The X-ray emissivities are from the VVAPEC model (Smith et al. 2001) using AtomDB version 2.0.2, as implemented in XSpec (Arnaud 1996) version 12.0.9c. The wind opacities are from Verner \& Yakovlev (1995) (obtained using the interface of Leutenegger et al. 2010), and the interstellar medium (ISM) opacities are from the TBabs model (Wilms et al. 2000). As the emissivities and wind opacities are metallicity dependent, we use three models to cover the range of WR spectral types in the SPH simulations; one for the WC stars (Crowther 2007), one for WN5-7 (Onifer et al. 2008), and one for WN8-9 and Ofpe/WN9 (CMFGEN website). Given that the high ISM column density obscures $E<1 \mathrm{keV}$, the optical depths over the observable range are sufficiently low such that the radiative transfer is well into the optically thin limit. 


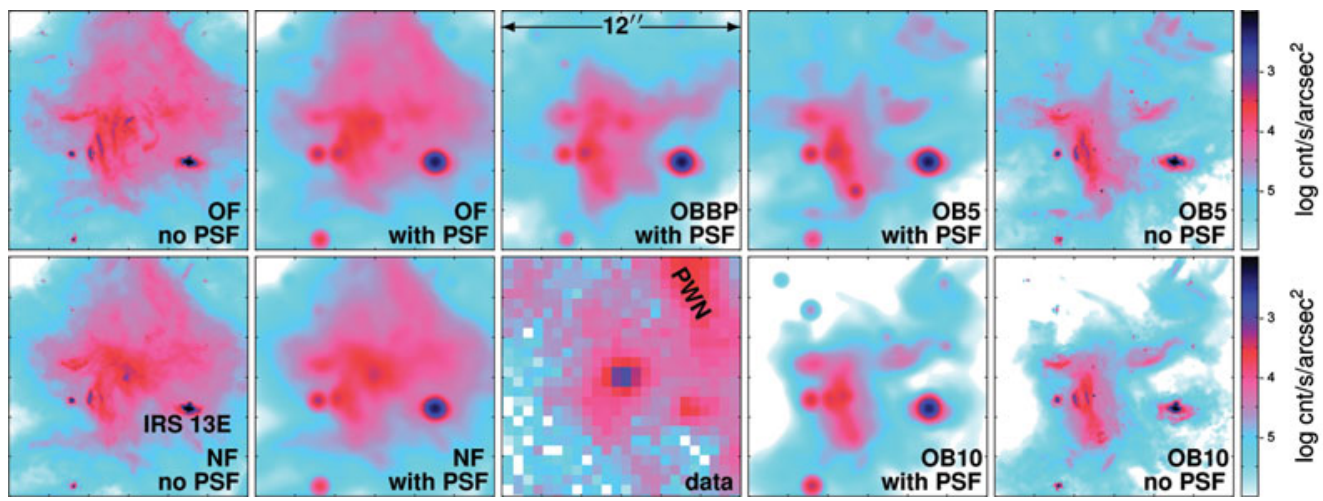

Figure 2. Chandra 4-9 keV ACIS-S/HETG 0th-order images $\left(12^{\prime \prime} \times 12^{\prime \prime}\right)$ comparing all models with the observation, which is in the bottom centre panel. For the models, the first and last columns are not folded through the PSF, while the 3 central columns are. The SMBH feedback strength increases clockwise (NF to OB10), while the X-ray flux decreases, showing that the clearing out of material by the SMBH feedback (Fig. 1) affects the thermal X-ray emission.
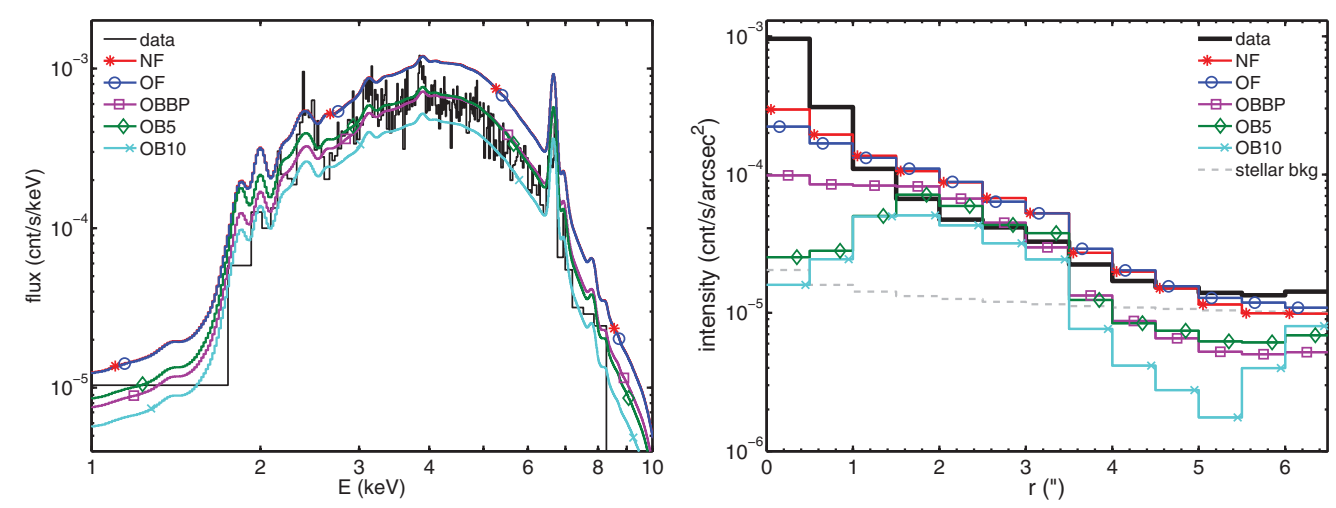

Figure 3. Left: Chandra ACIS-S/HETG 0th-order spectra of the models and observation from the $2^{\prime \prime}-5^{\prime \prime}$ ring centred on Sgr A* (excluding the IRS $13 \mathrm{E}$ and PWN contributions). As expected, the level of X-ray flux follows the trend of Fig 2. Right: 4-9 keV intensity of the observation and models as a function of radius from $\mathrm{Sgr} \mathrm{A}^{*}$. The background component due to the stellar population of CVs, which has been subtracted from the observational image, is shown.

The only free parameter in the model is the ISM absorbing column, $N_{\mathrm{H}}$. Table 1 (center) shows the fitting results for each model obtained with XSpec. The best-fit value is $N_{\mathrm{H}}=1.1 \times 10^{23} \mathrm{~cm}^{-2}$, which we use to make all X-ray images and spectra in this paper.

\section{Results}

Figs. $2 \& 3$ (left) show the $4-9 \mathrm{keV}$ images and the $2^{\prime \prime}-5^{\prime \prime}$ ring spectra for all models. To compare the model and data images more quantitatively, Fig. 3 (right) shows the 4-9 $\mathrm{keV}$ intensity as a function of projected radius from $\mathrm{Sgr} \mathrm{A}^{*}$.

A noteworthy achievement of the model is the agreement in the spectral shape (Fig. 3, left). This provides strong evidence that the WR wind-wind collisions are the dominant source of X-ray emission around Sgr A*. The temperature of the gas around Sgr A* is naturally explained by the WR shocked material, validating the wind speeds of the WR stars used in the simulations. 
The model that best agrees with the observation over $2^{\prime \prime}-5^{\prime \prime}$ (excluding IRS $13 \mathrm{E}$ and the PWN) is the medium-strength feedback model OB5 (see Table 1, right). The ratio of the model-to-data flux over $4-9 \mathrm{keV}$ is 0.92 and 1.20 in the image and spectral comparison, yielding a mean discrepancy of the model flux being $\sim 6 \%$ higher than the data.

The largest disagreement is the IRS $13 \mathrm{E}$ cluster (model/data $\sim 7$ ), indicating that its WC and WN winds should be revised downward to agree with the X-ray observations.

\section{Conclusions}

We compute the thermal X-ray properties of the Galactic centre from hydrodynamic simulations of the $30 \mathrm{WR}$ stars orbiting within $12^{\prime \prime}$ of Sgr A* (Cuadra et al. 2008, 2015). These simulations use different feedback models from the SMBH at its centre. The Chandra X-ray Visionary Program observations (Wang et al. 2013) provide an anchor point for these simulations, so we compare the observed $12^{\prime \prime} \times 12^{\prime \prime} 4-9 \mathrm{keV}$ image and the $2^{\prime \prime}-5^{\prime \prime}$ ring spectrum with the same observables synthesized from the models. Remarkably, the shape of the model spectra, regardless of the type of feedback, agree very well with the data. This indicates the hot gas around $\mathrm{Sgr} \mathrm{A}^{*}$ is primarily from shocked WR wind material, and that the velocities of these winds are well constrained. The X-ray flux strongly depends on the feedback mechanism; greater SMBH outflows clear out more WR-ejected material around Sgr A*, thus decreasing the model X-ray emission. Over 4-9 keV in energy and $2^{\prime \prime}-5^{\prime \prime}$ in projected distance from Sgr A* (excluding IRS 13E and the nearby $\mathrm{PWN}$ ), the X-ray emission from all models is within a factor of 2 of the observations, with the best model agreeing to within $10 \%$; this is the medium-strength feedback model OB5, which has an SMBH outburst of $\dot{M}_{\text {out }}=10^{-4} M_{\odot} \mathrm{yr}^{-1}$ and $v=5000 \mathrm{~km} \mathrm{~s}^{-1}$ from 400 to $100 \mathrm{yr}$ ago. Therefore, this work shows that the SMBH outburst is required for fitting the X-ray data, and by extension that the outburst still affects the current X-ray emission around Sgr A*, even though it ended 100 yr ago.

\section{References}

Arnaud, K. A. 1996, Astronomical Data Analysis Software and Systems V, ASP Conference Series, 101, 17

Blandford, R. D. \& Begelman, M. C. 1999, MNRAS (Letters), 303, L1

Crowther, P. A. 2007, ARAA, 45, 177

Cuadra, J., Nayakshin, S., \& Martins, F. 2008, MNRAS, 383, 458

Cuadra, J., Nayakshin, S., \& Wang, Q. D. 2015, MNRAS, 450, 277

Leutenegger, M. A., Cohen, D. H., Zsargó, J., Martell, E. M., MacArthur, J. P., Owocki, S. P., Gagné, M., \& Hillier, D. J. 2010, ApJ, 719, 1767

Onifer, A., Heger, A., \& Abdallah, J. 2008, The Metallicity Dependence of Wolf-Rayet Mass Loss, ASP Conference Series, 391, 305

Ponti, G., Terrier, R., Goldwurm, A., Belanger, G., \& Trap, G. 2010, ApJ, 714, 732

Price, D. J. 2007, PASA, 24, 159

Russell, C. M. P. 2013, Ph.D. thesis, Univ. Delaware

Russell, C. M. P., Corcoran, M. F., Hamaguchi, K., Madura, T. I., Owocki, S. P., \& Hillier, D. J. 2016, MNRAS, 458, 2275

Russell, C. M. P., Wang, Q. D., \& Cuadra, J. 2016, MNRAS, in press, arXiv:1607.01562

Smith, R. K., Brickhouse, N. S., Liedahl, D. A., \& Raymond, J. C. 2001, ApJ Lett., 556, L91

Verner, D. A. \& Yakovlev, D. G. 1995, A\&AS, 109, 125

Wang, Q. D., Nowak, M. A., Markoff, S. B., Baganoff, F. K., Nayakshin, S., Yuan, F., Cuadra, J., Davis, J., Dexter, J., Fabian, A. C., Grosso, N., Haggard, D., Houck, J., Ji, L., Li, Z., Neilsen, J., Porquet, D., Ripple, F., \& Shcherbakov, R. V. 2013, Science, 341, 981

Wilms, J., Allen, A., \& McCray, R. 2000, ApJ, 542, 914 